\title{
Feasibility of zero or near zero fluoroscopy during catheter ablation procedures
}

\author{
Laurent M. Haegeli ${ }^{1,2}$ * , Linda Stutz ${ }^{1, *}$, Mohammed Mohsen ${ }^{1}$, Thomas Wolber ${ }^{1}$, \\ Corinna Brunckhorst ${ }^{1}$, Chol-Jun On ${ }^{1}$, Firat Duru ${ }^{1}$ \\ ${ }^{1}$ Arrhythmia and Electrophysiology, Department of Cardiology, University Heart Center, \\ University Hospital Zurich, Switzerland \\ ${ }^{2}$ Division of Cardiology, Medical University Department, Kantonsspital Aarau, Switzerland
}

\begin{abstract}
Background: Awareness of risks associated with radiation exposure to patients and medical staff has significantly increased. It has been reported before that the use of advanced three-dimensional electroanatomical mapping (EAM) system significantly reduces fuoroscopy time, however this study aimed for zero or near zero fluoroscopy ablation to assess its feasibility and safety in ablation of atrial fibrillation $(A F)$ and other tachyarrhythmias in a "real world" experience of a single tertiary care center.

Methods: This was a single-center study where ablation procedures were attempted without fluoroscopy in 34 consecutive patients with different tachyarrhythmias under the support of EAM system. When transseptal puncture (TSP) was needed, it was attempted under the guidance of intracardiac echocardiography (ICE).

Results: Among 34 patients consecutively enrolled in this study, 28 (82.4\%) patients were referred for radiofrequency ablation (RFA) of $A F, 3(8.8 \%)$ patients for ablation of right ventricular outflow tract (RVOT) ventricular extrasystole (VES), 1 (2.9\%) patient for ablation of atrioventricular nodal reentry tachycardia (AVNRT), 2 (5.9\%) patients for typical atrial futter ablation. In 21 (62\%) patients the entire procedure was carried out without the use of fluoroscopy. Among $28 \mathrm{AF}$ patients, 15 (54\%) patients underwent ablation without the use of fluoroscopy and among these 15 patients, 10 (67\%) patients required TSP under ICE guidance while 5 (33\%) patients the catheters were introduced to left atrium through a patent foramen ovale. In 13 AF patients, fluoroscopy was only required for double TSP. The total procedure time of $A F$ ablation was $130 \pm 50 \mathrm{~min}$. All patients referred for atrial flutter, AVNRT, and VES of the RVOT ablation did not require any fluoroscopy.

Conclusions: This study demonstrates the feasibility of zero or near zero fluoroscopy procedure including TSP with the support of EAM and ICE guidance in a "real world" experience of a single tertiary care center. When fluoroscopy was required, it was limited to TSP hence keeping the radiation dose very low. (Cardiol J 2019; 26, 3: 226-232)
\end{abstract}

Key words: catheter ablation, fluoroscopy, atrial fibrillation, arrhythmia, three-dimensional electroanatomical mapping

\section{Introduction}

Recently, the awareness of risks associated with radiation exposure to patients and medical staff has significantly increased. The risks of injury hazard by medical radiation exposure are caused by deterministic (dose-dependent) effects such as radiation injury to the skin secondary to high peak skin doses and by stochastic (non dose-dependent) effects such as increased radiation-induced cancer

Address for correspondence: Laurent M. Haegeli, MD, Arrhythmia and Electrophysiology, Department of Cardiology, University Heart Center, University Hospital Zurich, Zurich, Switzerland, tel: +41 442558762 , fax: +41 442554401 , e-mail: laurent.haegeli@usz.ch

Received: 22.07.2017 Accepted: 22.01.2018

*Laurent M. Haegeli and Linda Stutz share first authorship. 
risk and genetic effects [1,2]. These risks are of particular concern for young or obese patients and for patients who undergo long, repeated or complex procedures [3, 4]. Moreover, operators, nurses and technical staff are exposed to significant and potentially hazardous doses of ionizing radiation on a daily basis resulting in life-time risk for malignancy, cataract and congenital defects [5, 6]. Since the beginning of interventional electrophysiology for diagnosis and therapy of cardiac arrhythmias, fluoroscopy techniques have been widely used to navigate catheters within the heart and the vessels and to monitor their position. In recent years, technical developments have led to the routine and wide-spread use of non-fluoroscopic threedimensional (3D) electro-anatomical navigation and mapping systems for radiofrequency ablation (RFA) of tachyarrhythmias including atrial fibrillation (AF) resulting in a significant reduction of radiation dose during mapping and ablation of complex arrhythmias [7-13]. Minimizing the exposure to radiation is recommended by the American College of Cardiology teaching the ALARA principle - keeping the radiation "as low as reasonably achievable" $[14,15]$. It has been reported before that the use of 3D electro-anatomical mapping (EAM) system significantly reduces fluoroscopy time and radiation dose however the aim of this study was to assess the feasibility and the safety of zero or near zero fluoroscopy mapping and ablation of AF and other tachyarrhythmias in a "real world" experience of a single tertiary care center.

\section{Methods}

Among patients referred to the Division of Arrhythmia and Electrophysiology at the University Heart Center in Zurich, Switzerland, 34 patients were enrolled consecutively in the study. Majority of patients in the study group were referred for RFA of AF (28 patients). Few patients with other tachyarrhythmias were also enrolled: 2 with typical atrial flutter (AFL), 3 with idiopathic ventricular extrasystole (VES) from the right ventricular outflow tract (RVOT) and 1 with atrioventricular nodal reentry tachycardia (AVNRT). Percutaneous access for all catheters was via the right femoral vein. Written informed consent was obtained from all patients. All clinical cardiac characteristics as well as important comorbidities were recorded according to the regulations of the local Institutional Committee on Human Research. This was a single-center study.

\section{Procedural safety}

Pre-procedure and post-procedure patient management, as well as intra-procedure anticoagulation policy were in accordance with practice guidelines and our hospital policies.

All operators performing the ablation procedures were senior staff with experience in catheter navigation under 3D EAM system. Different imaging modalities were used including intracardiac echocardiography (ICE), transesophageal echocardiography (TEE) and magnetic resonance imaging (MRI) as needed. Although the intention was to perform zero fluoroscopy procedure, all operators were allowed to use fluoroscopy if required for patient safety and that is why fluoroscopy was used in 13 patients.

\section{Catheter ablation of AF}

Patients scheduled for AF ablation underwent MRI of the pulmonary veins (PV) and left atrium (LA) before the procedure to identify anatomical variants, to assess the exact anatomical position and size of PV ostia and to merge with CARTO 3 system (Biosense Webster, Diamond Bar, CA, USA) (Fig. 1). TEE was also done before the procedure to rule out thrombi in the LA and to document the presence of a patent foramen ovale (PFO). For mapping and ablation, a 3D EAM system, CARTO 3 system (Biosense Webster, Diamond Bar, CA, USA) was used. All procedures were performed under conscious sedation using midazolam and fentanyl.

A 6 F steerable decapolar Dynamic XT catheter (Boston Scientific, Marlborough, MA, USA) was positioned in the coronary sinus for mapping and pacing purposes. For ablation an $8 \mathrm{~F}$ Thermocool bi-directional (D-F curve) catheter was used (Biosense Webster, Diamond Bar, CA, USA). A steerable $7 \mathrm{~F}$ multipolar Lasso catheter (Biosense Webster, Diamond Bar, CA, USA) was used for mapping of the PVs and to confirm procedural endpoint of electrical isolation. The transseptal punctures (TSP) were performed using Brockenborough (BRK-1) needle (St. Jude Medical, St. Paul, MN, USA) through 8 F Preface guiding sheath (Biosense Webster, Diamond Bar, CA, USA) and was attempted under the guidance of Acuson Acuna ultrasound catheter (Siemens Healthcare, Erlangen, Germany).

Catheter positioning into and within the cardiac chamber of interest as well as mapping and ablation were attempted without fluoroscopy with the support of EMA. A fast anatomical map was created 


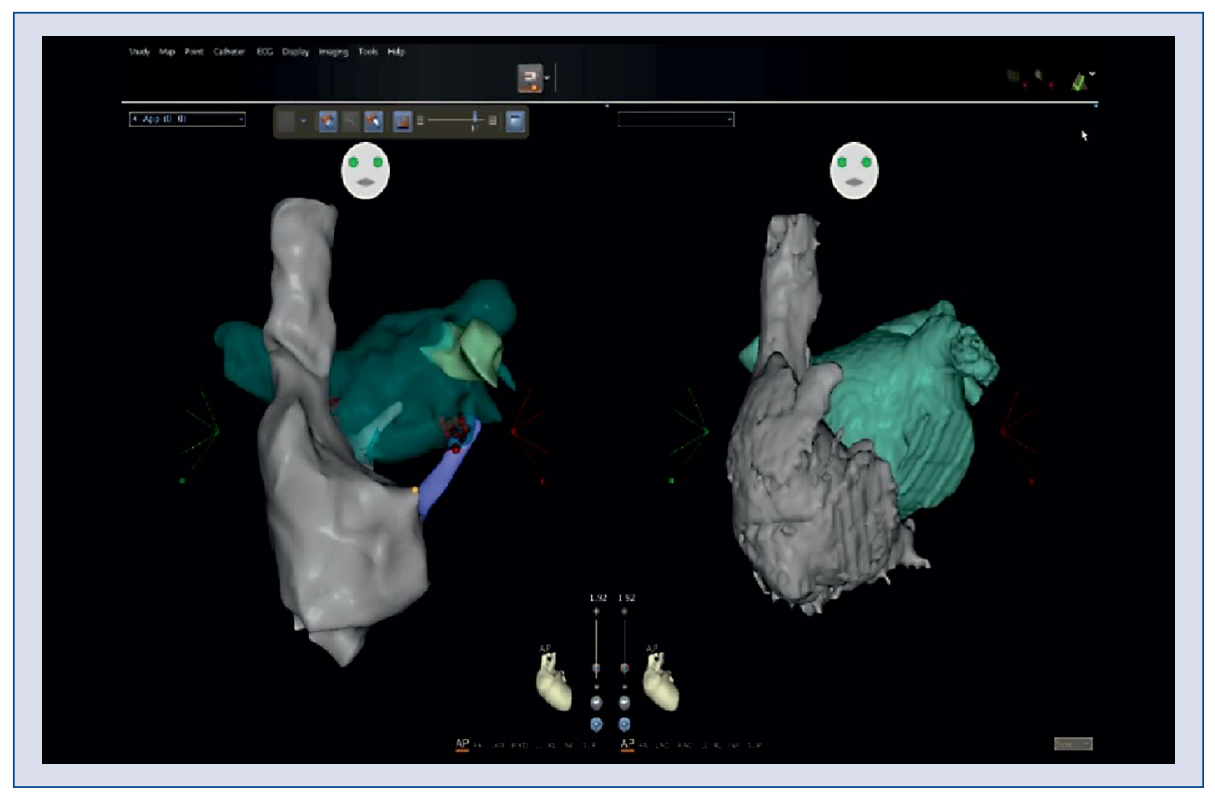

Figure 1. Left panel showing the three-dimensional electroanatomical CARTO maps (Biosense Webster, Diamond Bar, CA, USA) of right (gray) and left (green) atria with coronary sinus (blue) and right panel with three-dimensional reconstruction from magnetic resonance imaging. Both panels show the images from the anteroposterior view.

using the Lasso catheter to create a 3D geometry of LA and PV without fluoroscopy. PV isolation was performed by wide-area circumferential point-bypoint RFA around the ostia of the ipsilateral PVs. The procedural endpoint was electrical isolation (entrance and exit block) documented using the Lasso mapping catheter. Power was limited to 30 Watts and flow was adjusted from $2 \mathrm{~mL} / \mathrm{min}$ up to $30 \mathrm{~mL} / \mathrm{min}$ to achieve this power. In patients with a history of typical AFL (5 patients in our group) a cavotricuspid isthmus ablation line was added. If sinus rhythm was not restored following ablation, the patient was cardioverted with a direct current shock.

In 5 patients the catheters were introduced to LA through a PFO without the need of TSP. In 3 patients with PFO, ICE was used to allocate the site of PFO that was crossed by the ablation catheter along with its Preface guiding sheath. In 2 patients with PFO, the ablation catheter (through the Preface guiding sheath) was brought to the superior vena cava and dragged caudally till the typical jump to the foramen ovale region was detected with 3D EAM. Then the ablation catheter along with its guiding sheath were advanced into the LA.

In 1 patient, TSP was done without the use of fluoroscopy or ICE as following: first a fast anatomical map was created using the ablation catheter (through Preface guiding sheath) to cre- ate a $3 \mathrm{D}$ right atrium and vena cava geometry. The His bundle region was marked. Then the Preface guiding sheath (with only the tip of ablation catheter exposed out of the sheath) was brought to the superior vena cava and dragged caudally till the typical jump to the foramen ovale region was detected and marked with 3D EAM. The ablation catheter was pulled out of the guiding sheath and the sheath was kept in same place of the foramen ovale region. The dilator of the guiding sheath and the Brockenbrough (BRK-1) needle (with metal arrow on needle hub and sheath sidearm pointing at 4 o'clock) were inserted within the sheath and TSP was done successfully.

\section{Catheter ablation of RVOT VES}

Thermocool bi-directional (D-F curve) catheter was used (Biosense Webster, Diamond Bar, CA, USA) to create RVOT geometry. Earliest electrical activation region was mapped in 3D EAM system and VES was successfully ablated.

\section{Catheter ablation of typical AFL}

A 6 F steerable decapolar Dynamic XT catheter (Boston Scientific, Marlborough, MA, USA) was positioned in the coronary sinus. Thermocool bi-directional (D-F curve) catheter was used (Biosense Webster, Diamond Bar, CA, USA) to create right atrium geometry with the tricuspid annulus and both vena cava superior and inferior. The His 
bundle region was marked. An ablation line was performed in the region of cavotricuspid isthmus creating a bi-directional block.

\section{Catheter ablation of AVNRT}

A Navistar $4 \mathrm{~mm}$ (D Curve) ablation catheter (Biosense Webster, Diamond Bar, CA, USA) was used to create right atrium geometry. The His bundle region was marked and the anatomical and characteristical electrical slow pathway region was identified and successfully ablated.

\section{Results}

Thirty four patients were consecutively enrolled in the study. The baseline clinical characteristics are summarized in Table 1. All patients had a structurally normal heart with preserved left ventricular systolic function and without valvular heart disease. Twenty eight $(82.4 \%)$ patients were referred for catheter ablation of AF (22 with paroxysmal and 6 with persistent AF). The remaining 6 patients were referred either for ablation of typical AFL, VES from the RVOT or AVNRT (Table 2). Among AF patients, 5 patients had additional typical AFL. $39 \%$ of AF patients had anatomical variants of the PVs and $18 \%$ had a PFO (Table 3 ).

In $21(62 \%)$ patients the entire procedure was carried out without the use of fluoroscopy. Among the $28 \mathrm{AF}$ patients, the procedure was performed with zero fluoroscopy in $15(54 \%)$ patients. Ten (67\%) AF patients required TSP under ICE guidance to access the LA while in $5(33 \%)$ patients the catheters were introduced into the LA through a PFO (in 3 patients with the help of ICE and in 2 without as previously described). All patients in the series referred for atrial AFL, AVNRT, and VES of the RVOT ablation did not require fluoroscopy (Fig. 2).

\section{Procedure time and fluoroscopy use}

The total procedure time for ablation of $\mathrm{AF}$ was $130 \pm 50 \mathrm{~min}$. Most of the time $(59.9 \pm 20 \mathrm{~min})$ was required for the ablation of PVs (Table 4). Fluoroscopy was required in $13 \mathrm{AF}$ patients solely for double TSP in order to safely access the LA despite the availability of ICE. In our patient series fluoroscopy was only used for the TSPs. The median total fluoroscopy time was $2.6 \pm 2.2 \mathrm{~min}$ and the dose area product $13 \pm 27$ gray $\times \mathrm{cm}^{2}$. Mapping of the LA and wide-area circumferential ablation around the PVs did not require the use of fluoroscopy.
Table 1. Patient characteristics.

\begin{tabular}{lc}
\hline Number of patients & $34(100 \%)$ \\
Age [years] & $56 \pm 12.4$ \\
Male & $24(71 \%)$ \\
Left atrium diameter [mm] & $41.7 \pm 0.6$ \\
Hypertension & $15(44.1 \%)$ \\
Coronary artery disease & $6(17.7 \%)$ \\
Diabetes mellitus & $1(2.9 \%)$ \\
CVA/TIA & $5(14.7 \%)$ \\
Heart failure & $2(5.9 \%)$ \\
\hline
\end{tabular}

CVA - cerebrovascular attack; TIA — transient ischemic attack

Table 2. Types of arrhythmias.

\begin{tabular}{lc}
\hline Atrial fibrillation: & $28(82.4 \%)$ \\
Paroxysmal AF & 22 \\
Persistent AF & 6 \\
Atrial flutter: & \\
Atrial flutter alone & $2(5.9 \%)$ \\
In combination with paroxysmal AF & $4(11.8 \%)$ \\
In combination with persistent AF & $1(2.9 \%)$ \\
VES RVOT & $3(8.8 \%)$ \\
AVNRT & $1(2.9 \%)$ \\
\hline
\end{tabular}

AF — atrial fibrillation; VES - ventricular extrasystole; RVOT right ventricular outflow tract; AVNRT — atrioventricular reentrant tachycardia

Table 3. Pulmonary vein anatomy.

\begin{tabular}{ll}
\hline Normal anatomy (2 right, & $17(61.0 \%)$ \\
2 left pulmonary veins) & \\
Common left ostium & $6(21.4 \%)$ \\
Three right pulmonary veins & $5(17.9 \%)$ \\
Patent foramen ovale & $5(17.9 \%)$ \\
\hline
\end{tabular}

Table 4. Procedural and fluoroscopy times.

\begin{tabular}{lc}
\hline Total procedure time [min] & $130 \pm 50$ \\
Catheter placement [min] & $20 \pm 5$ \\
Left atrial mapping [min] & $15 \pm 8$ \\
Left atrial ablation [min] & $59.9 \pm 20$ \\
Total X-ray time [min] & $2.6 \pm 2.2$ \\
Dose area product [Gray $\times \mathrm{cm}^{2}$ ] & $13 \pm 27$ \\
\hline
\end{tabular}

\section{Procedural endpoint and outcome}

All procedures were successfully carried out. In AF patients, isolation of all PVs was validated using the Lasso catheter placed at each ostium 


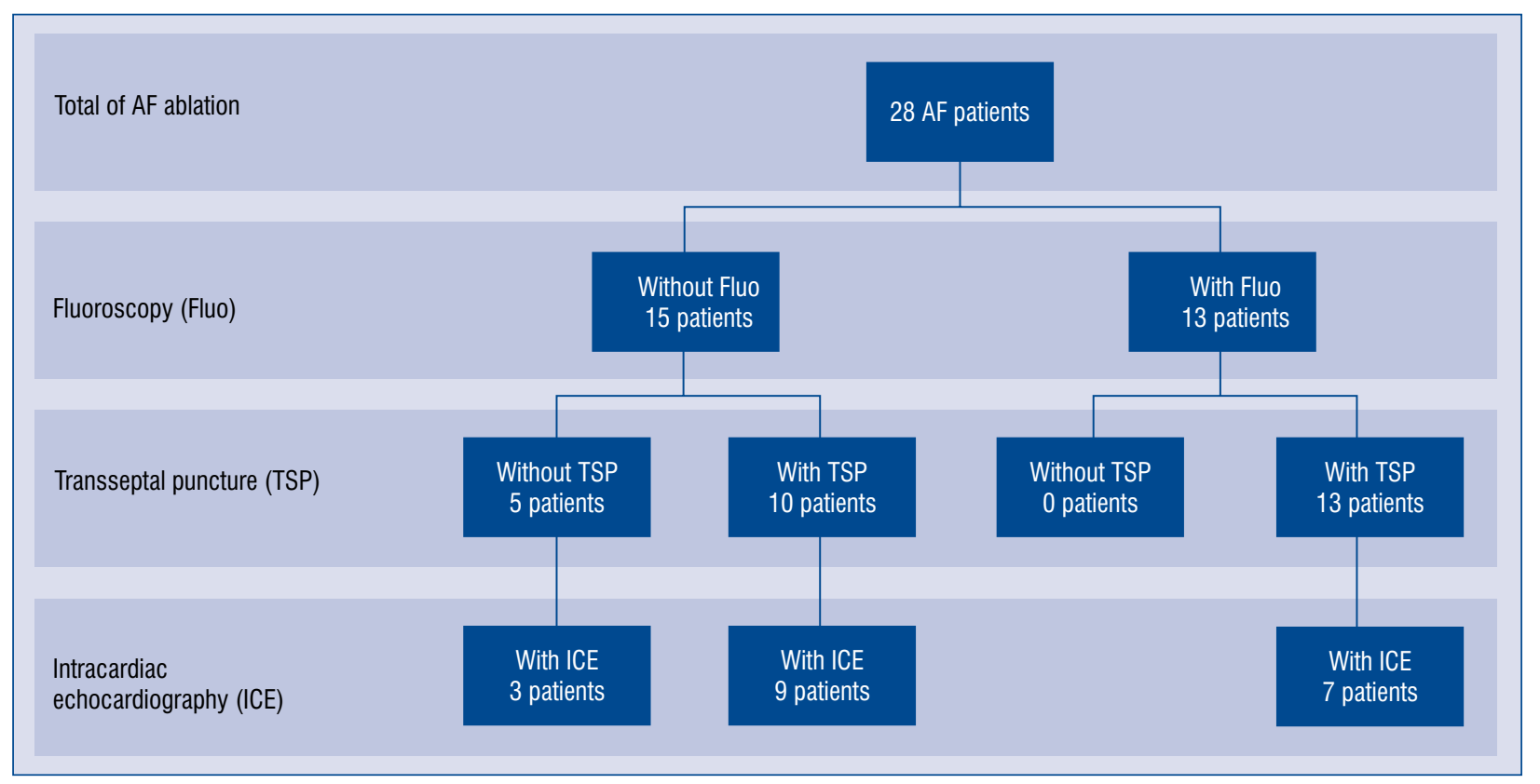

Figure 2. Atrial fibrillation (AF) ablation and fluoroscopy use.

which realized confirmation of isolation of all PVs by documentation of entrance and exit block. For typical AFL ablation, the cavotricuspid isthmus was checked for bidirectional block. VES of the RVOT was ablated successfully with complete abolition of VES. After slow pathway ablation, there was no AVNRT inducible at rest or under adrenergic stimulation with isoprenalin.

One patient in whom AF ablation was performed without fluoroscopy and after uncomplicated TSP guided by ICE had a post-procedural pericardial tamponade which was resolved by immediate percutaneous drainage. No other complications occurred in this study group.

\section{Discussion}

Over the last years, the awareness of radiation hazards to patients and professional staff has significantly increased. Reduction of radiation exposure in electrophysiological procedures, such as AF ablation, should always be considered. 3D EAM systems with their constant updating technologies that allows precise location visualization of diagnostic and ablation catheters and allows fast anatomical mapping of cardiac chambers have shown to contribute to significant reduction of fluoroscopy time and radiation dose. Lee et al. [16] have shown that the use of contact forcesensing catheter during AF ablation significantly reduces fluoroscopy times by $77 \%$, radiation dose by $71 \%$, and procedural time by $19 \%$. However the median fluoroscopy time in the force-sensing catheter group was $9.5 \mathrm{~min}$ and median radiation dose was $1043.5 \mathrm{mGy} \times \mathrm{cm}^{2}$. This study showed that zero or near zero fluoroscopy was feasible in patients referred for catheter ablation of $\mathrm{AF}$ and other tachyarrhythmias with the support of EAM and ICE guidance. Fluoroscopy was only used if required and limited to assist in difficult TSP hence keeping the radiation time and dose very low $\left(2.6 \pm 2.2\right.$ min and $13 \pm 2.7$ gray $\times \mathrm{cm}^{2}$, respectively). Catheter placement, navigation, mapping and ablation can be done with the support of EAM. TSP can be done safely under the guidance of ICE imaging. The LA can be accessed safely in the presence of PFO under ICE guidance without the need of TSP.

Other groups have shown feasibility of fluoroscopy-free PV isolation in 26 out of 30 consecutive patients with a documented PFO [17]. Bulava et al. [18] demonstrated the feasibility of zero fluoroscopy ablation in 40 patients with paroxysmal AF using ICE imaging and 3D EAM mapping with contact force measurements. Reddy et al. [19] showed complete fluoroless catheter ablation of paroxysmal AF in 20 patients using combination of ICE and 3D EAM system.

In a series of 19 out of 21 patients, Ferguson et al. [20] demonstrated that AF ablation was feasible without fluoroscopic guidance. Double TSP were performed with ICE guidance and facilitated by electrocautery. In 2 cases fluoroscopy was used to assist TSP. 
Kerst et al. [21] showed in a group of 30 patients that contact force-controlled zero fluoroscopy catheter ablation is generally feasible in right-sided and left atrial cardiac arrhythmias.

Hindricks et al. [22] compared the results of catheter ablation to cure typical AFL using conventional ablation strategy and electro-anatomically guided mapping and ablation. They found that cavotricuspid isthmus ablation to cure typical AFL was highly effective and safe, both in conventional and electro-anatomically guided ablation group. The use of EAM system significantly reduced fluoroscopy exposure time by almost $50 \%$, however this was at the expense of an increased cost of the procedure.

\section{Limitations of the study}

Because of the small number of patients in the resent study group, it was difficult to make conclusions about safety. A much larger multi-center study would be needed to determine any true increase in the rate of catheter-related complications as a result of using this technique given that the incidence of cardiac tamponade and catheter perforation during $\mathrm{AF}$ ablation is low.

\section{Conclusions}

This study demonstrates the feasibility of an entire zero or near zero fluoroscopy procedure including transseptal puncture for patients with $\mathrm{AF}$ and other tachyarrhythmias under the support of 3D EAM systems and ICE imaging in a "real world" experience of a single tertiary care center. When fluoroscopy was required, it was limited to transseptal puncture and hence keeping the radiation dose very low.

Conflict of interest: Laurent M. Haegeli reports compensation for participation on a speaker bureau from St. Jude Medical and Biosense Webster. Other authors have no disclosures.

\section{References}

1. Lickfett L, Mahesh M, Vasamreddy C, et al. Radiation exposure during catheter ablation of atrial fibrillation. Circulation. 2004; 110(19): 3003-3010, doi: 10.1161/01.CIR.0000146952.49223.11, indexed in Pubmed: 15505084.

2. Rosenthal LS, Beck TJ, Williams J, et al. Acute radiation dermatitis following radiofrequency catheter ablation of atrioventricular nodal reentrant tachycardia. Pacing Clin Electrophysiol. 1997; 20(7): 1834-1839, indexed in Pubmed: 9249839.

3. Ector J, Dragusin O, Adriaenssens B, et al. Obesity is a major determinant of radiation dose in patients undergoing pulmonary vein isolation for atrial fibrillation. J Am Coll Cardiol. 2007; 50(3): 234-242, doi: 10.1016/j.jacc.2007.03.040, indexed in Pubmed: 17631216.

4. Kidouchi T, Suzuki S, Furui S, et al. Entrance skin dose during radiofrequency catheter ablation for tachyarrhythmia: a multicenter study. Pacing Clin Electrophysiol. 2011; 34(5): 563-570, doi: 10.1111/j.1540-8159.2010.03016.x, indexed in Pubmed: 21609338.

5. Calkins H, Niklason L, Sousa J, et al. Radiation exposure during radiofrequency catheter ablation of accessory atrioventricular connections. Circulation. 1991; 84(6): 2376-2382, indexed in Pubmed: 1959193.

6. Perisinakis K, Damilakis J, Theocharopoulos N, et al. Accurate assessment of patient effective radiation dose and associated detriment risk from radiofrequency catheter ablation procedures. Circulation. 2001; 104(1): 58-62, indexed in Pubmed: 11435338.

7. Scaglione M, Biasco L, Caponi D, et al. Visualization of multiple catheters with electroanatomical mapping reduces X-ray exposure during atrial fibrillation ablation. Europace. 2011; 13(7): 955-962, doi: 10.1093/europace/eur062, indexed in Pubmed: 21421574 .

8. Stabile G, Scaglione M, del Greco M, et al. Reduced fluoroscopy exposure during ablation of atrial fibrillation using a novel electroanatomical navigation system: a multicentre experience. Europace. 2012; 14(1): 60-65, doi: 10.1093/europace/eur271, indexed in Pubmed: 21893511.

9. Huo Y, Christoph M, Forkmann M, et al. Reduction of radiation exposure during atrial fibrillation ablation using a novel fluoroscopy image integrated 3-dimensional electroanatomic mapping system: A prospective, randomized, single-blind, and controlled study. Heart Rhythm. 2015; 12(9): 1945-1955, doi: 10.1016/j. hrthm.2015.05.018, indexed in Pubmed: 26001508.

10. Steven D, Servatius H, Rostock T, et al. Reduced fluoroscopy during atrial fibrillation ablation: benefits of robotic guided navigation. J Cardiovasc Electrophysiol. 2010; 21(1): 6-12, doi: 10.1111/j.1540-8167.2009.01592.x, indexed in Pubmed: 19793149.

11. Raju H, Whitaker J, Taylor C, et al. Electroanatomic mapping and transoesophageal echocardiography for near zero fluoroscopy during complex left atrial ablation. Heart Lung Circ. 2016; 25(7): 652-660, doi: 10.1016/j.hlc.2016.01.018, indexed in Pubmed: 26979468.

12. Khaykin Y, Oosthuizen R, Zarnett L, et al. CARTO-guided vs. NavX-guided pulmonary vein antrum isolation and pulmonary vein antrum isolation performed without 3-D mapping: effect of the 3-D mapping system on procedure duration and fluoroscopy time. J Interv Card Electrophysiol. 2011; 30(3): 233-240, doi: 10.1007/s10840-010-9538-9, indexed in Pubmed: 21253840.

13. Kozluk E, Rodkiewicz D, Piątkowska A, et al. Safety and efficacy of cryoablation without the use of fluoroscopy. Cardiol J. 2018; 25(3): 327-332, doi: 10.5603/CJ.a2017.0065, indexed in Pubmed: 28612907.

14. Limacher MC, Douglas PS, Germano G, et al. ACC expert consensus document. Radiation safety in the practice of cardiology. American College of Cardiology. J Am Coll Cardiol. 1998; 31(4): 892-913, indexed in Pubmed: 9525565.

15. Haegeli LM, Calkins H. Catheter ablation of atrial fibrillation: an update. Eur Heart J. 2014; 35(36): 2454-2459, doi: 10.1093/ eurheartj/ehu291, indexed in Pubmed: 25053659.

16. Lee G, Hunter RJ, Lovell MJ, et al. Use of a contact force-sensing ablation catheter with advanced catheter location significantly re- 
Cardiology Journal 2019, Vol. 26, No. 3

duces fluoroscopy time and radiation dose in catheter ablation of atrial fibrillation. Europace. 2016; 18(2): 211-218, doi: 10.1093/ europace/euv186, indexed in Pubmed: 26318548.

17. Kühne M, Knecht S, Mühl A, et al. Fluoroscopy-Free pulmonary vein isolation in patients with atrial fibrillation and a patent foramen ovale using solely an electroanatomic mapping system. PLoS One. 2016; 11(1): e0148059, doi: 10.1371/journal. pone.0148059, indexed in Pubmed: 26820309.

18. Bulava A, Hanis J, Eisenberger M. Catheter ablation of atrial fibrillation using zero-fluoroscopy technique: a randomized trial. Pacing Clin Electrophysiol. 2015; 38(7): 797-806, doi: 10.1111/ pace.12634, indexed in Pubmed: 25790320.

19. Reddy VY, Morales G, Ahmed H, et al. Catheter ablation of atrial fibrillation without the use of fluoroscopy. Heart Rhythm. 2010; 7(11): 1644-1653, doi: 10.1016/j.hrthm.2010.07.011, indexed in Pubmed: 20637313.
20. Ferguson JD, Helms A, Mangrum JM, et al. Catheter ablation of atrial fibrillation without fluoroscopy using intracardiac echocardiography and electroanatomic mapping. Circ Arrhythm Electrophysiol. 2009; 2(6): 611-619, doi: 10.1161/CIRCEP.109.872093, indexed in Pubmed: 20009075.

21. Kerst G, Weig HJ, Weretka S, et al. Contact force-controlled zero-fluoroscopy catheter ablation of right-sided and left atrial arrhythmia substrates. Heart Rhythm. 2012; 9(5): 709-714, doi: 10.1016/j.hrthm.2011.12.025, indexed in Pubmed: 22222276.

22. Hindricks G, Willems S, Kautzner J, et al. EuroFlutter Investigators. Effect of electroanatomically guided versus conventional catheter ablation of typical atrial flutter on the fluoroscopy time and resource use: a prospective randomized multicenter study. J Cardiovasc Electrophysiol. 2009; 20(7): 734-740, doi: 10.1111/j.1540-8167.2009.01439.x, indexed in Pubmed: 19298568. 